\title{
Inferring network connectivity using kinetic Ising models
}

\author{
John A Hertz ${ }^{1,2^{*}}$, Yasser Roudi ${ }^{2}$, Andreas Thorning ${ }^{1}$, Joanna Tyrcha ${ }^{3}$, Erik Aurell ${ }^{4,5}$, Hong-Li Zeng ${ }^{6}$ \\ From Nineteenth Annual Computational Neuroscience Meeting: CNS*2010 \\ San Antonio, TX, USA. 24-30 July 2010
}

One approach that has been explored recently for analyzing functional connectivity involves parametrizing the spike pattern distribution by an Ising model with symmetric connectivity. However, the connections found using this procedure do not generally agree well with the true synaptic connectivity [1]. Here we try, instead, a kinetic Ising network with asymmetric connections [2], updated either asynchronously or synchronously. For these models, it is possible to derive an iterative algorithm for the optimal connection strengths by formally maximizing the log-likelihood of the measured history [2]. For weak coupling, one can expand to first order and derive an approximate closed-form expression for the connections that takes the form $\mathbf{J}=\mathbf{A D C}^{-1}$, where $\mathbf{C}$ is the equal-time correlation matrix, $\mathbf{D}$ is the correlation matrix with time lag one step, and $\mathrm{A}$ is a diagonal matrix with elements $A_{i i}=1 /\left(1-\left\langle S_{i}\right\rangle^{2}\right)$.

We have tested the iterative algorithm on data generated by randomly-connected, synchronously-updated Ising networks for a range of sizes, firing rates, noise levels and concentration of nonzero connections. In all cases, the rms reconstruction error falls off approximately like a $-1 / 3$ power of the length of the run used to generate the correlations statistics. The approximate formula gives qualitatively good results, enabling us to identify the strongest connection reliably, though the magnitudes obtained tend to be off by a scaling factor that depends on noise level and mean firing rate. These conclusions hold for the asynchronous model as well. Furthermore, if the inference is done on a subset of the neurons in the network, the connections among them can still be recovered approximately. This approximation becomes excellent in the limit of weak connection concentration, where it permits reliable identification of the nonzero connections.
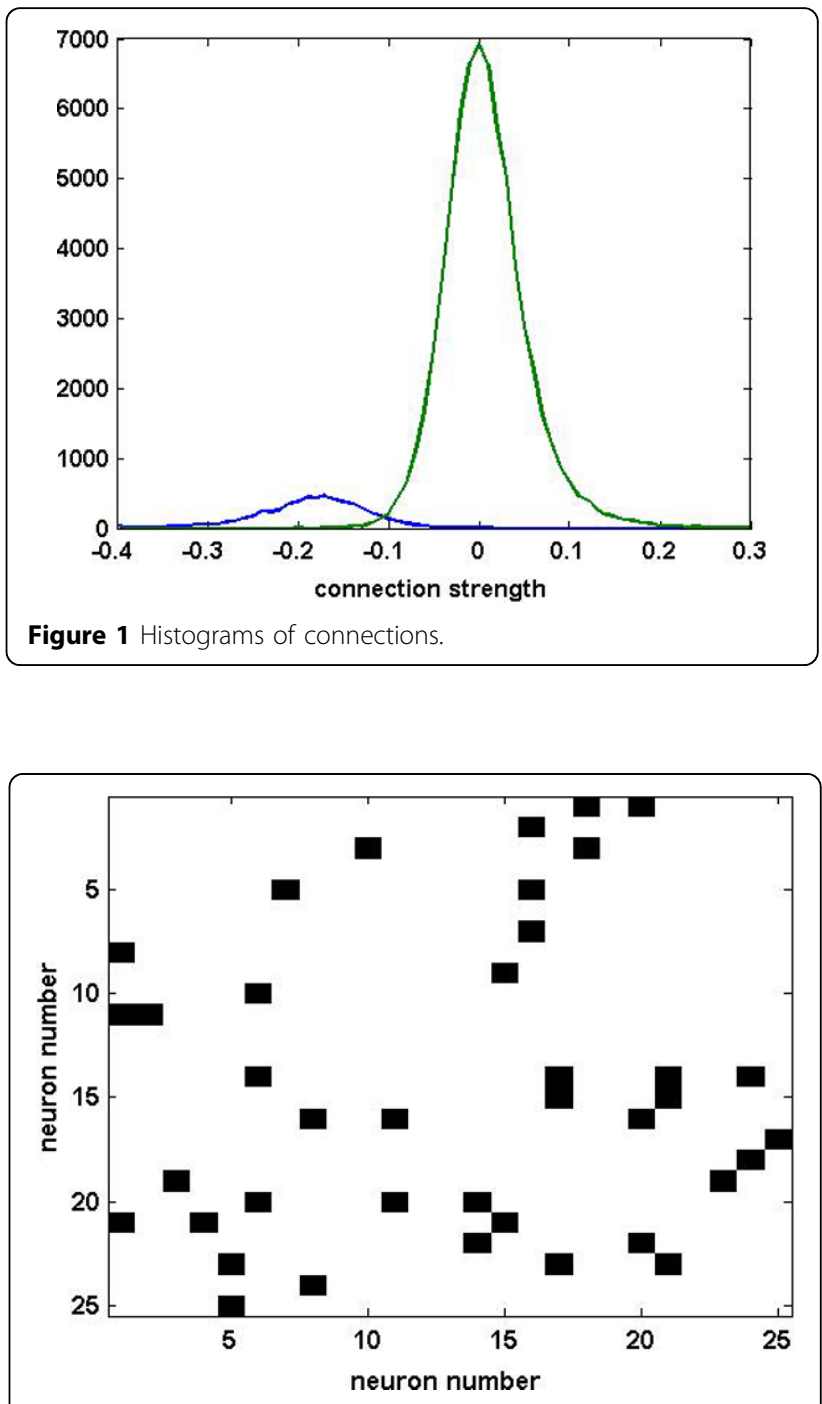

Figure 2 Connections in model.

\footnotetext{
* Correspondence: hertz@nbi.dk

${ }^{1}$ Niels Bohr Institute, Copenhagen University, 2100 Copenhagen $\varnothing$, Denmark
} 


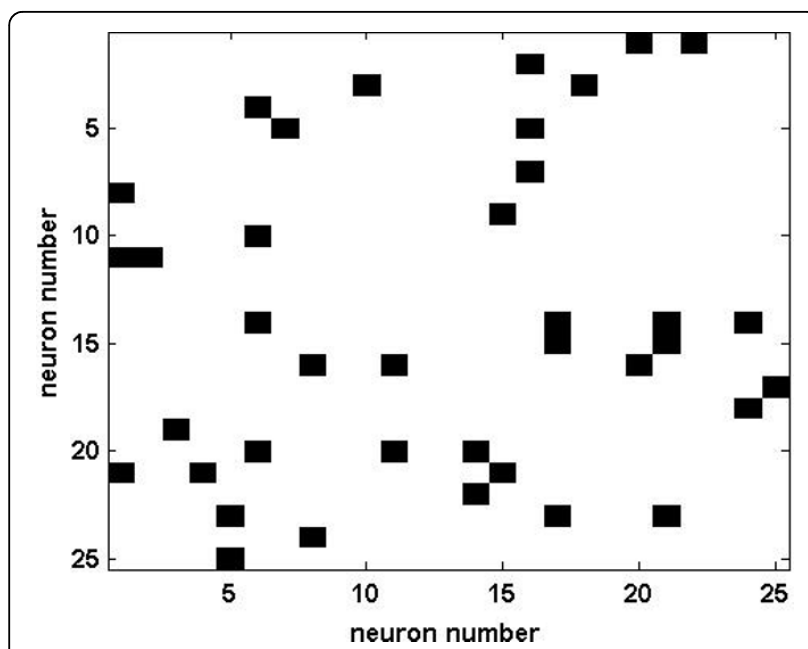

Figure 3 Inferred connections.

We applied the approximate formula to data from a realistic cortical network model [3]. Fig. 1 shows histograms of the connection strengths found in 30 randomly chosen sets of $n=50$ inhibitory neurons, separated according to whether there actually was a synapse connecting the pair in question (blue) or not (green). If the $c n(n-1)$ pairs with the most negative inferred couplings are identified as connected, with $c$ the connection probability in the population, we find average false-positive and false-negative rates of $5.6 \%$ and $7.2 \%$, respectively. To illustrate the point visually, Fig. 2 shows the actual connections in the original simulated network and Fig. 3 shows those identified by this procedure for a set of 25 neurons.

\section{Author details}

${ }^{1}$ Niels Bohr Institute, Copenhagen University, 2100 Copenhagen $\varnothing$, Denmark. ${ }^{2}$ Nordita, 10691 Stockholm, Sweden. ${ }^{3}$ Institute of Mathematics, Stockholm University, 10691 Stockholm, Sweden. ${ }^{4}$ Department of Computational Biology, Royal Institute of Technology, 10691 Stockholm, Sweden. ${ }^{5}$ Department of Information and Computer Science, Helsinki University of Technology, 02015 TKK Espoo, Finland. 'Department of Applied Physics, Helsinki University of Technology, 02015 TKK Espoo, Finland.

Published: 20 July 2010

\section{References}

1. Roudi Y, Tyrcha J, Hertz J: Ising model for neural data: model quality and approximate methods for extracting functional connectivity. Phys Rev $E$ 2009, 79:051915.

2. Roudi Y, Aurell E, Hertz J: Statistical physics of pairwise probability models. Frontiers Comput Neurosci 2009, 3:22.

3. Hertz J: Cross-correlations in high-conductance states of a model cortical network. Neural Comp 2010, 22(2):427-447.

\section{Submit your next manuscript to BioMed Central} and take full advantage of:

- Convenient online submission

- Thorough peer review

- No space constraints or color figure charges

- Immediate publication on acceptance

- Inclusion in PubMed, CAS, Scopus and Google Scholar

- Research which is freely available for redistribution

Submit your manuscript at www.biomedcentral.com/submit 